effort of seeking active treatment from a pharmacy may be unwilling to accept anything else. The appropriateness and feasibility of such an approach have yet to be tested. Comparative studies should present no such problems.

Blinding is one way of excluding potential bias. One pharmacy trial chose not to blind because it would require the participation of the general practitioner. ${ }^{9}$ The European Commission's guidelines for good clinical practice specify that the person responsible for the trial should be a doctor or dentist, though some customers of pharmacies may be reluctant to accept notification to their general practitioner and his or her involvement as part of the protocol. The value of a non-blinded study of this type is limited, particularly when patients and investigators assess the results without objective measurements.

Complete blinding, with double dummy techniques to overcome differences in formulation, may be unrealistic in a community pharmacy setting, and single blinding should be sufficient if only patients' assessments are used. There seems no reason why potential subjects cannot be persuaded of the need to judge a medicine free of preconceptions, knowing that it is not a placebo. Those who do not accept blinding should not be excluded completely but included in a non-blinded arm, which could be assessed separately for possible bias and could add further useful data on safety. Blinded trials in the community pharmacy require further evaluation.

A thornier problem is defining criteria of symptoms for admission and assessment. Standardisation with appropriate protocols is essential: people attend pharmacies complaining of vague problems, which, for a clinical trial, must be defined. Definitions of diarrhoea - of both frequency and severityvary. How runny is a runny nose? How bad is a headache?
Assessments by both the pharmacist and customer are therefore important. Once admitted to the study, subjects are perfectly capable of assessing their own symptoms with visual analogue scales and symptom diaries. For example, one comparison of cold symptoms by both doctors and subjects at the Medical Research Council's Common Cold Unit recorded a close correlation between the two ${ }^{10}$ suggesting that this methodology is valid.

If health policy is to encourage the public to use community pharmacies pharmacists will need information on the relative efficacy and safety of over the counter drugs. The role of pharmacy based clinical trials is potentially valuable and worthy of further testing.

Drug Information Specialist,

STEVE CHAPLIN

Haydon Bridge,

Northumberland NE47 6HG

Director of Pharmacy Postgraduate Studies,

Centre for Pharmacy Postgraduate Medical Education,

University of Manchester, Manchester M13 9PL

1 Quick JM, Blenkinsopp A. A community pharmacy study to compare the control of hay fever symptoms by astemizole $10 \mathrm{mg}$ and terfenadine $120 \mathrm{mg}$. Pharmaceutical fournal 1991;246:272-5. Department of Health. Promoting better health. London: HMSO, 1987.

3 Smith FJ, Salkind MR. Factors influencing the pharmacist's advisory role in Greater London. Pharmaceutical fournal 1990;244(suppl): R4-7.

Cartwright A, Anderson R. Patients and their doctors revisited. London: Tavistock Press, 1981. Royal College of Physicians of London. Research involving patients. London: Royal College of Physicians, 1990.

Group Working Party on Efficacy of Medicinal Products. Notes for guidance. Brussels: Commission of the European Communities, 1988.

Milburn LJ, Dunbar PE, Kendall HE. Factors influencing pharmacy practice in the West Midlands. Pharmaceutical fournal 1990;245:R19.

Brich CR. Pralley C. Palfour preferen:245:R19.

9 Noyelle RM, Crossland DM, Pickvance NJ. munity study. Pharmaceutical foumal 1987;238:561-4.

10 Macintyre S, Pritchard C. Comparisons between the self-assessed and observer-assessed presence and severity of colds. Soc Sci Med 1989;29:1243-8.

\title{
Psychiatric symptoms and low blood pressure
}

\section{More evidence for an association}

German doctors apparently have available some 85 preparations for treating low blood pressure (defined as systolic pressure $<110 \mathrm{~mm} \mathrm{Hg}$ or diastolic pressure $<60 \mathrm{~mm} \mathrm{Hg}$ ). German medical textbooks attribute symptoms such as mental and physical fatigue, dizziness, depression, and anxiety to hypotension, ${ }^{12}$ for which one in 20 German women is being treated. Similarly, a survey of French speaking Canadians found that one in 10 of those attending for blood pressure screening was already being treated for hypotension. ${ }^{3}$

The Anglophone medical community currently takes a different view. Hypotension is recognised in its acute stage, after haemorrhage or myocardial infarction; in a chronic form, resulting from persisting cardiac damage or Addison's disease; and as orthostatic hypotension. ${ }^{+}$Constitutional hypotension, however, is not recognised as a disease, being variously described as a "non-disease," "rarely symptomatic and treatment not indicated," and the "ideal normal blood pressure." Attention has focused on an apparent excess of psychiatric symptoms associated with hypertension, although such excess is probably secondary to the diagnosis and labelling of the hypertensive state or to treatment with antihypertensive drugs.

How ironic, therefore, that at the time when Britain is so preoccupied with Europeanisation, analysis of data from the first phase of the second Whitehall study found a relation between low systolic blood pressure and complaints of tiredness and responses to a valid measure of psychiatric morbidity (the general health questionnaire) (p 75). ${ }^{8}$ The association between low blood pressure and complaints of tiredness, however, disappeared in an analysis that controlled for the questionnaire's score. This is the second paper published in this journal within a year to show the presence of symptomatic hypotension in a British population. An analysis of data from the health and lifestyle survey also showed that complaints of tiredness and feeling faint were increasingly common with lower levels of blood pressure. ${ }^{9}$ In contrast to the Whitehall study, however, in the health and lifestyle survey the score on the general health questionnaire did not show any relationship with blood pressure. Both analyses carefully controlled for evident confounders of age, sex, body mass, drugs, smoking, current physical illness, and current level of exercise.

What might explain these associations? A critic might comment on the inconsistency between the studies and ask whether minor psychiatric morbidity or fatigue was the main consequence of hypotension. The two are not necessarily the same. Secondly, as the data were collected for other purposes an unknown confounder is possible. The analyses were carried out on the data from $74 \%$ and $82 \%$ of the respondents, respectively, so a response bias could, therefore, have operated. But an explanation parallel to that in hypertension 
- that the psychiatric symptoms are secondary to being labelled-is unlikely, given that low blood pressure in the United Kingdom is likely to be a cause for felicitation. Methodological limitations must be considered, but the collection of data by observers who were uninterested in this association at the time, and the strength of the associations reported, means that the findings cannot be easily dismissed.

Neither sets of authors speculate on the cause of this association. What could they be? Could hypoperfusion impair cerebral monoamine activity? Could hypoperfusion lead to tissues, particularly muscle, being deprived of an adequate oxygen supply, causing the symptoms of fatigue ${ }^{20}$ Could the presence of depression or anxiety, from whatever cause, be associated with a persistent change in vasomotor tone? Evidence to support these speculations is lacking. Another option is to regard both as manifestations of interindividual variation, characterised by certain psychological responses (fatigue or depression) on the one hand and physiological state (persistent hypotension) on the other. (Another example of linked psychological responses and physiological states is the increased and delayed hypertensive response to certain stresses observed in people with type A behaviour. ${ }^{11}$ ) Such a constitution was, in fact, recognised earlier this century,,$^{12}$ and the neurasthenic patient has always been said to have lower than average blood pressure.

Should anything be done now? Keeping a very open mind is important. It could be that the Anglophone medical community has been working with a blinkered assumption that as high blood pressure is "bad" for the patient so low blood pressure must be "good." It may be that psychiatric morbidity modestly impairing the quality of life, associated with low blood pressure, has been overlooked. Secondly, it seems worth while to explore evidence that antihypotensive regimens help such morbidity. Clinical trials could be considered in collaboration with German or French colleagues. It is time for British doctors to put their Euroscepticism to the test.

Professor of Epidemiological Psychiatry and Vice Dean,

ANTHONY MANN

Institute of Psychiatry,

London SE5 8AF

1 Payer L. Medicine and culture: varieties of treatment in the United States, England, West Germany and France. New York: Henry Holt, 1988.

2 Pemberton J. Does constitutional hypotension exist? BMf 1989;298:660-2.

3 Robbins JM, Korda $\mathrm{H}$, Shapiro MF. Treatment for a nondisease: the case of low blood pressure. Soc Sci Med 1982;16:27-33.

4 Harrison TR. Principles of internal medicine. New York: McGraw Hill, 1987

5 Tonkin A, Wing L. Hypotension: assessment and management. Med f Aust 1990;153:474-85.

6 Robinson S. Hypotension: the ideal normal blood pressure. N Engl f Med 1940;233:407-16.

7 Mann A. Hypertension: psychological aspects and diagnostic impact in a clinical trial. Psychol Med Monogr Suppl 1984;5:1-35.

Milgrim J, Stansfeld S, Marmot M. Low blood pressure, low mood? BMF 1992;304:75-8.
P.

8 Pilgrim J, Stansfeld S, Marmot M. Low blood pressure, low mood? BMf 1992;304:75-8. 1990;301:362-5.

10 Simpson LO. Symptoms of low blood pressure. BMY 1990;301:815

11 Manuck SB, Craft S, Gold KJ. Coronary-prone behaviour pattern ard cardiovascular disease. Psychophysiology 1978;15:403-11.

12 Dally J. Low blood pressure: its causes and significance. London: Heinemann, 192

\section{Guillain-Barré syndrome revisited}

\section{Pathogenesis still unknown}

When Guillain, Barré, and Strohl made their original observations on two patients with progressive weakness, areflexia, and a high concentration of protein in their cerebrospinal fluid' ${ }^{1}$ there was heated debate over the relation of their syndrome with its good prognosis to the more serious and fatal case of ascending paralysis that Landry had described in detail some 60 years before. ${ }^{2}$ Subsequent authors have taken a broad view of the syndrome, which has come to be known as the Guillain-Barré syndrome, and have used the term to encompass several different clinical presentations and outcomes.

Diagnostic criteria for the Guillain-Barré syndrome are wide and select a group of acute idiopathic neuropathies on the basis of progressive limb weakness and areflexia without evidence of porphyria or exposure to lead or toxins. Mild sensory signs, lack of fever, raised concentrations of protein in the cerebrospinal fluid, and neurophysiological evidence of demyelination are all helpful but not essential for the diagnosis. There remains no laboratory test to identify the disease reliably. A prospective study of the syndrome's outcome found that the median time to complete functional recovery was nine months, but one in five patients were left unable to work at 12 months and a further one in eight had died. ${ }^{3}$ Poor outcome is associated with more rapidly progressive disease, the need for assisted ventilation, the presence of small distally evoked muscle action potentials, and older age. Antecedent infections with numerous agents have been reported, but convincing controlled data are available only for campylobacter, cytomegalovirus, and Epstein-Barr virus. ${ }^{+}$ The nature of the antecedent infection has little effect on the clinical features or outcome with the exception of campylobacter, which correlates with poor outcome, perhaps reflecting a different pathogenesis. About $3 \%$ of patients with the acute disease develop a chronic or relapsing course. These patients differ in having a higher prevalence of certain HLAs (CW7 and B8) and responding to immunosuppression. ${ }^{5}$

Necropsy studies suggest that different pathological processes recur within the syndrome, contributing to the variation in clinical features and outcome. A perivascular inflammatory infiltrate was prominent in 12 cases in an influential study of the syndrome in $1969^{6}$ and came to be regarded as the pathological hallmark of the disease. Later reports have suggested that lymphocytic infiltration is prominent in only some cases and many show demyelination without obvious lymphocytes. ${ }^{7}$ A few very severe cases that fulfil diagnostic criteria for Guillain-Barré syndrome have pathological evidence of an axonal neuropathy. ${ }^{8}$ Such patients have peripheral nerves that are difficult to stimulate electrically and recover poorly.

How infection triggers the disease is unknown. The generation of autoimmune responses because of similarities in structure between infective agents and host proteins is the most favoured hypothesis, but hard evidence is lacking. It is tempting to speculate that the pathogenesis of cases of Guillain-Barré syndrome with appreciable lymphocytic infiltration is mediated by $\mathrm{T}$ cells while those with few lymphocytes present are mediated by antibodies. Efforts to show cellular immune hypersensitivity to neural antigens in patients with Guillain-Barré syndrome have been disappointing despite evidence supporting their role in experimental allergic neuritis in animal models.

Lymphocytes from the blood of a few patients respond to the P2 protein of myelin. ${ }^{9}$ Complement fixing antibodies to neural antigens have been found occasionally by many authors but have been described in more than three quarters 